\title{
Evaluación del riesgo de extinción de Mammillaria pectinifera, cactácea endémica de la región de Tehuacán-Cuicatlán
}

\author{
Assessment of extinction risk of Mammillaria pectinifera, an endemic cactus of the Tehuacán- \\ Cuicatlán region
}

Pedro Luis Valverde*, José Alejandro Zavala-Hurtado, Cecilia Jiménez-Sierra, Beatriz Rendón-Aguilar, Amelia Cornejo-Romero, Sombra Rivas-Arancibia, Gerardo López-Ortega y Marco Aurelio Pérez-Hernández

Departamento de Biología, Universidad Autónoma Metropolitana-Iztapalapa, Apartado postal 55-535, 09340 México, D. F., México

*Correspondencia: plvp@xanum.uam.mx

Resumen. Mammillaria pectinifera (Cactaceae) es una especie endémica de la Región de Tehuacán-Cuicatlán en los estados de Puebla y Oaxaca. Aunque en la Norma Oficial Mexicana NOM-059-ECOL-2001 está considerada como amenazada y está incluida en el Apéndice I de la CITES, la información derivada de los escasos estudios ecológicos sobre esta especie revela serios riesgos de extinción de sus poblaciones. En el presente estudio se registra una evaluación del riesgo de extinción de M. pectinifera, para la que se empleó el protocolo del Método de Evaluación del Riesgo de Extinción de las Especies Silvestres en México (MER) del Anexo I de la NOM059-ECOL-2001, el cual se basa en 4 criterios para evaluar cualitativamente el riesgo de extinción de cualquier especie silvestre en el territorio nacional. Dicha evaluación se apoya en datos de campo de 7 poblaciones e información procedente de diversas fuentes. De acuerdo con los 12 puntos obtenidos del MER, se propone que $M$. pectinifera se considere en la categoría de especie en peligro de extinción (P) en la NOM-059-ECOL-2001 y sea incluida de nuevo en la Lista Roja de la UICN. Finalmente se plantea una propuesta para mejorar la valoración del Criterio D del MER.

Palabras clave: disturbio, distribución, especie rara, MER, vulnerabilidad biológica.

\begin{abstract}
Mammillaria pectinifera (Cactaceae) is an endemic species from the Tehuacán-Cuicatlán region at the Mexican States of Puebla and Oaxaca. Although it is considered as a threatened species under Mexican law NOM-059-ECOL-2001, and it is already included in the CITES Appendix I, ecological studies reveal serious vulnerability to extinction of its populations. In this study, we conducted an extinction risk assessment for M. pectinifera based on the protocol of the Method for Evaluation of Risk of Extinction for Mexican Wild Species (MER) from Appendix I of NOM-059-ECOL-2001, which is based on a qualitative assessment of 4 criteria for evaluating extinction risk of any wild species in the Mexican territory. The present assessment relies on data from field studies on 7 populations as well as information from several sources. The MER assessment resulted in a risk score of 12 points and, accordingly, we propose to consider M. pectinifera as a species under extinction risk (P) in NOM-059-ECOL-2001. Also, it should be included again in the Red List of IUCN. Finally, we suggest ways for improving the assessment of Criterion D of the MER.
\end{abstract}

Kew words: disturbance, distribution, rare species, MER, vulnerability.

\section{Introducción}

Un alto número de especies de la familia de las cactáceas se encuentra en riesgo de extinción (Hunt, 1992; Anderson et al., 1994; Hernández y Godínez, 1994; GodínezÁlvarez et al., 2003), como es el caso de Mammillaria pectinifera (Stein) F.A.C.Weber in Bois, una pequeña cactácea globosa solitaria de ca. $3 \mathrm{~cm}$ de diámetro (Fig. 1), endémica de la Región de Tehuacán-Cuicatlán en los estados de Puebla y Oaxaca (Meyrán, 1973; Bravo-Hollis,

Recibido: 21 enero 2008; aceptado: 27 junio 2008
1978; Bravo-Hollis y Sánchez-Mejorada, 1991). Muy apreciada por los coleccionistas desde su descubrimiento a finales del siglo XIX, $M$ pectinifera fue descrita como Pelecyphora pectinata por Stein en 1885; posteriormente, nombrada Pelecyphora aselliformis var. pectinifera Hort ex Rümpler y en 1898 descrita como Mammillaria con el nombre especifico pectinifera (Glass, 1998).

Si bien, en la actualidad se encuentra señalada como especie amenazada (A) en la Norma Oficial Mexicana NOM-059-ECOL-2001 (SEMARNAT, 2002) y es un taxón incluido en el Apéndice I de la CITES (Hunt, 1992; Hernández y Godínez, 1994; Glass, 1998; Guzmán et al., 2003; Arias et al., 2005; CITES, 2007), la información 


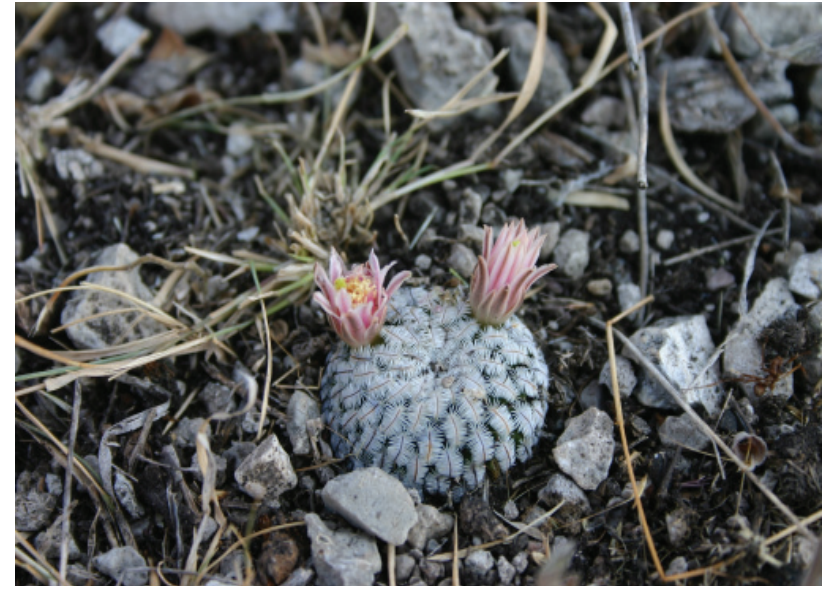

Figura 1. Individuo de Mammillaria pectinifera en Texcala, Puebla.

sobre $M$. pectinifera en los escasos estudios ecológicos revela que sus poblaciones presentan serios riesgos de extinción, debido principalmente a la colecta intensiva para fines comerciales y a la degradación del hábitat donde se desarrolla (Glass, 1998; Martorell y Peters, 2005). El objetivo del presente estudio es evaluar el riesgo de extinción de las poblaciones silvestres de $M$. pectinifera mediante la aplicación del Método de Evaluación del Riesgo de Extinción de las Especies Silvestres en México (MER), para actualizar su estatus de conservación en la Norma Oficial Mexicana NOM-059-ECOL-2001 (SEMARNAT, 2002).

La Norma Oficial Mexicana tiene como fin identificar las especies o poblaciones de flora y fauna silvestres en riesgo en la República Mexicana para integrar las listas correspondientes y se establecen los criterios para incluir, excluir o cambiar la categoría de riesgo de las especies o poblaciones, mediante un método de evaluación del riesgo de extinción (SEMARNAT, 2002). Este protocolo conocido como "Método de Evaluación del Riesgo de Extinción de las Especies Silvestres en México" (MER; SEMARNAT, 2002) tiene como finalidad unificar los criterios para asignar las siguientes categorías de riesgo de extinción de cualquier especie silvestre en México (Tambutti et al., 2001): sujeta a protección especial (Pr), amenazada (A) o en peligro de extinción (P) (SEMARNAT, 2002). Aunque algunos aspectos del MER son vulnerables y subjetivos, este método facilita la toma de decisiones y permite la generación de hipótesis a probar en futuros estudios (Olson et al., 2005).

Área de estudio. La Reserva de la Biosfera TehuacánCuicatlán se encuentra en la región sureste del estado de Puebla y noreste de Oaxaca. En la zona domina un ambiente semiárido con la presencia de matorrales xerófilos (Vite et al., 1992; Zavala-Hurtado y Hernández-Cárdenas, 1998).
El clima es cálido semi-desértico $\left(\mathrm{BS}_{0} \mathrm{hw} "(\mathrm{w})(\mathrm{e})(\mathrm{g})\right.$ de acuerdo con la clasificación de Köppen, modificada por García, 1973); la temperatura media anual es de $21^{\circ} \mathrm{C}$ y la precipitación media anual es de $380 \mathrm{~mm}$. La mayor parte de las lluvias se presentan en el verano (mayo y junio) y otoño (septiembre) (García, 1973).

La región se distingue por un alto grado de diversidad biológica y de endemismos, los cuales parecen haber sido favorecidos tanto por la posición fitogeográfica del área, su relieve y sus condiciones climáticas, así como por el aislamiento geográfico de la zona, el cual podría haberse originado desde el Plioceno cuando apareció el Eje Neovolcánico Transversal (Rzedowski, 1988). En esta zona, la familia Cactaceae ocupa el cuarto lugar por el número de géneros que presenta (21) e incluye al menos 15 especies endémicas (Dávila et al., 1995).

\section{Materiales y métodos}

El estatus de riesgo determinado a través del MER se basa en la valoración de cuatro criterios que abajo se describen, divididos a su vez en distintos valores numéricos ascendentes que reflejan la magnitud del mismo (Tambutti et al., 2001). Así, los valores más altos para cada criterio corresponden a condiciones poco propicias para la preservación de la especie. La suma total establece un valor numérico con el cual se determina la categoría de riesgo de la especie: sujeta a protección especial (Pr) (menos de 10 puntos); amenazada (A) (de 10 a 11 puntos); o en peligro de extinción (P) (de 12 a 14 puntos) (SEMARNAT, 2002). El MER fue diseñado para incorporar la información disponible, de muy diversa naturaleza y calidad, y traducirla en los distintos puntajes requeridos por sus cuatro criterios (Tambutti et al., 2001). La evaluación presentada aquí se apoya, tanto en datos propios obtenidos durante estudios de campo, como en información procedente de artículos científicos, informes técnicos y ejemplares de herbario. Los 4 criterios de riesgo, sus categorías y los métodos para estimar los puntajes fueron los siguientes:

Criterio A) Amplitud de la distribución del taxón en México. Es el tamaño relativo del área de distribución del taxón respecto al área total del territorio nacional (ca. 2 millones de $\mathrm{km}^{2}$ ). Las especies con un área de distribución $<5 \%$ se consideran muy restringidas (valor de riesgo $=$ 4 ); entre el 5 y $15 \%$, restringidas (valor de riesgo $=3$ ); entre $15 \%$ y $40 \%$, medianamente restringidas o de amplia distribución (valor de riesgo $=2$ ) y con un área $>40 \%$, ampliamente distribuidas (valor de riesgo $=1$ ).

La amplitud de la distribución del taxón se determinó con base en recorridos de campo a las localidades conocidas por los autores, la revisión de la literatura y la 
consulta de información de ejemplares de herbario a través de las bases de datos disponibles en la Red Mundial de Información sobre Biodiversidad (REMIB: http://www. conabio.gob.mx/remib/doctos/remibnodosdb.html). Las poblaciones fueron ubicadas en un mapa (escala 1:600000) y se trazó el polígono de distribución. Adicionalmente, se determinó el aislamiento geográfico de las poblaciones con las distancias $(\mathrm{km})$ en línea recta entre ellas a partir de su ubicación en el mapa.

Criterio B) Estado del hábitat respecto al desarrollo natural del taxón. Se refiere a la condición actual del hábitat como un estimado del conjunto de requerimientos, tanto bióticos como abióticos, conocidos para el desarrollo natural del taxón. Los valores de riesgo son: hostil o muy limitante $(=3)$, intermedio o limitante $(=2)$ y propicio o poco limitante $(=1)$.

El estado del hábitat se caracterizó en cada localidad durante las visitas de campo (2005-2007) y por medio de la literatura. En cada localidad se registró altitud, pendiente y orientación del terreno, así como el tipo de vegetación. Mediante 3 transectos de $3 \times 30 \mathrm{~m}$ se registraron las especies vegetales dominantes, cobertura vegetal (\%), pedregosidad superficial (\%) y profundidad del suelo. Tanto la cobertura vegetal como la pedregosidad superficial se estimaron cualitativamente.

Criterio C) Vulnerabilidad biológica intrínseca del taxón. Considera todos aquellos atributos relacionados con la historia o forma de vida de una especie que la hacen vulnerable (e.g., estrategia reproductiva, demografía, fenología, especialización del hábitat y nodricismo). Los valores de riesgo son: vulnerabilidad alta $(=3)$, vulnerabilidad media $(=2)$ y vulnerabilidad baja $(=1)$.

En las poblaciones visitadas se emplearon de 3 a 15 transectos de $2 \times 30 \mathrm{~m}$ para estimar la densidad, así como el porcentaje de individuos bajo la protección de rocas $\mathrm{u}$ otras especies vegetales.

La descripción de la fenología, así como la estimación de la tasa de crecimiento y edad, se obtuvo con datos derivados de un estudio previo (1995-1996) en una población de la región dentro del estado de Puebla (San Antonio Texcala). En 6 cuadros permanentes $\left(1 \mathrm{~m}^{2}\right)$ se registró mensualmente para cada individuo el número de flores y frutos durante el periodo reproductivo. Se midió el diámetro de cada planta utilizando un vernier digital con resolución de $0.01 \mathrm{~mm}$ (Mitutoyo CD-S6). Estas medidas fueron tomadas cada 2 meses. Se estimó la tasa de crecimiento (mm/año) mediante un modelo de regresión entre el tamaño $(\mathrm{mm})$ y la tasa de crecimiento observada para los individuos que al menos sobrevivieron 6 meses después de iniciar las mediciones $(n=146)$. A partir de la tasa de crecimiento esperada se estimó la edad para las 4 categorías de tamaño $(\mathrm{mm})$ definidas por Valverde y
Zavala-Hurtado (2006): plántulas (0.01-2.50), juveniles (> 2.50-12.00), adultos pequeños ( $>12.00-20.00)$ y adultos grandes $(>20)$. Se obtuvo información adicional sobre la biología y ecología mediante observaciones realizadas durante las visitas a las localidades y consultando bases de datos electrónicas o bibliografía sobre $M$. pectinifera.

Criterio D) Impacto de la actividad humana sobre el taxón. Es una estimación numérica de la magnitud del impacto y las tendencias que generan las distintas actividades humanas sobre el taxón (e.g., proximidad a asentamientos humanos, fragmentación del hábitat, contaminación, realización de obras de infraestructura, uso, comercio y tráfico). Las categorías y valores de riesgo son: alto impacto $(=4)$, impacto medio $(=3)$ y bajo impacto $(=2)$.

Para evaluar el impacto de la actividad humana sobre M. pectinifera aplicamos el método propuesto por Martorell y Peters (2005) para obtener un índice de disturbio en cada una de las poblaciones estudiadas. Este es un índice multimétrico que mide la intensidad del disturbio basado en la cuantificación de 15 parámetros o indicadores (Martorell y Peters, 2005). En el presente trabajo se generó un nuevo índice a partir de las mediciones obtenidas durante los recorridos de campo con el objetivo de apoyar o modificar las conclusiones previamente obtenidas (Martorell y Peters 2005) respecto al efecto del disturbio sobre las poblaciones de esta especie.

Se construyó una matriz con 14 indicadores de 3 agentes de disturbio (ganadería, actividades humanas y deterioro del hábitat) medidos en cada una de las localidades visitadas (Cuadro 1). El indicador que se refiere a la densidad de caminos humanos se excluyó del análisis debido a que no fue posible estimarlo en todas las localidades. Se usaron tanto la definición como los 14 indicadores y la forma de evaluarlos desarrollados por Martorell y Peters (2005). Esta matriz fue estandarizada y sometida a un análisis de componentes principales (ACP) utilizando el paquete MVSP 3.12g (Kovach, 2004). A partir de la suma de los puntajes de los indicadores de cada agente para el primer componente extraído, se obtuvo un valor para cada uno de ellos. La suma de los valores de estos 3 agentes es igual al puntaje del ACP correspondiente a cada localidad y representa el valor del índice de disturbio (ID). Debido a que es frecuente obtener valores negativos en los puntajes del ACP, este índice se reescaló (ID ${ }_{\mathrm{r}}$ ) de tal manera que el valor más bajo del índice de disturbio tomara un valor de 0.0 y el más alto de 100.0.

La susceptibilidad al disturbio se evaluó analizando el efecto del grado de disturbio (ID) sobre la densidad de M. pectinifera en cada uno de los sitios estudiados (Peters y Martorell, 2000; Martorell y Peters, 2005). La relación entre el $\mathrm{ID}_{\mathrm{r}}$ y la densidad de $M$. pectinifera se analizó mediante una regresión log-lineal (Martorell y Peters, 
Cuadro 1. Agentes e indicadores de disturbio medidos en las 7 localidades de Mammillaria pectinifera evaluadas en este estudio siguiendo el método de Martorell y Peters (2005)

\begin{tabular}{|c|c|c|}
\hline Agentes & Indicadores & Abreviatura \\
\hline \multirow[t]{5}{*}{ Ganadería } & $\begin{array}{l}\text { Densidad de excretas de } \\
\text { cabra u oveja }\end{array}$ & CABR \\
\hline & $\begin{array}{l}\text { Densidad de excretas de } \\
\text { ganado mayor }\end{array}$ & GANA \\
\hline & $\begin{array}{l}\text { Fracción de plantas } \\
\text { ramoneadas }\end{array}$ & RAMO \\
\hline & Caminos ganaderos & CAGA \\
\hline & $\begin{array}{l}\text { Compactación del suelo por } \\
\text { ganado }\end{array}$ & COMP \\
\hline \multirow[t]{6}{*}{$\begin{array}{l}\text { Actividades } \\
\text { humanas }\end{array}$} & $\begin{array}{l}\text { Fracción de plantas } \\
\text { macheteadas }\end{array}$ & $\mathrm{MACH}$ \\
\hline & Evidencia de incendio & INCE \\
\hline & $\begin{array}{l}\text { Cobertura de caminos } \\
\text { humanos }\end{array}$ & $\mathrm{CCHU}$ \\
\hline & Cercanía de poblaciones & POBL \\
\hline & $\begin{array}{l}\text { Adyacencia a núcleos de } \\
\text { actividad }\end{array}$ & ADYA \\
\hline & Cambios de uso del suelo & USOS \\
\hline \multirow{3}{*}{$\begin{array}{l}\text { Deterioro } \\
\text { del hábitat }\end{array}$} & Erosión & EROS \\
\hline & Islas & ISLA \\
\hline & $\begin{array}{l}\text { Superficie totalmente } \\
\text { modificada }\end{array}$ & STOM \\
\hline
\end{tabular}

2005), ajustando a un modelo lineal generalizado, con una distribución Poisson y una función de enlace logarítmica (Crawley, 1996). Este análisis se realizó utilizando el paquete GLIM 4.0.

\section{Resultados}

Criterio A), amplitud de la distribución del taxón en México. Los recorridos de campo en las 7 localidades previamente identificadas como áreas de distribución de M. pectinifera, así como la información disponible en los herbarios y la literatura, permitieron ubicar 11 poblaciones (Cuadro 2) (Bravo-Hollis y Sánchez-Mejorada, 1991; Arias et al., 1997; Zavala-Hurtado, 1997; Peters y Martorell, 2000; Zavala-Hurtado y Valverde, 2003; Martorell y Peters, 2005). Aunque hay registros de hasta 19 localidades (Peters y Martorell, 2000), no fue posible obtener información acerca de las 8 restantes.

Sólo el Herbario Nacional de la Universidad Nacional Autónoma de México (MEXU; Colección Flora del Valle de Tehuacán-Cuicatlán, México, FES-I, UNAM) y el Real Jardín Botánico de Kew del Reino Unido (RBGKEW) cuentan con ejemplares de M. pectinifera. En MEXU existen 17 ejemplares y 1 en RBGKEW. Los 18
Cuadro 2. Localidades conocidas de Mammilaria pectinifera y área ocupada (Peters y Martorell, 2000)

\begin{tabular}{lcc}
\hline \multicolumn{1}{c}{ Localidad } & Estado & Área ocupada $\left(\mathrm{km}^{2}\right)$ \\
\hline Azumbilla & Puebla & 0.400 \\
Coapan $(*)$ & Puebla & 0.010 \\
El Riego $(*)$ & Puebla & 0.018 \\
Frontera $(*)$ & Oaxaca & 0.047 \\
Nicolás Bravo $(\bullet)$ & Puebla & - \\
Nopala & Puebla & 0.015 \\
Tecamachalco $(*)$ & Puebla & 0.135 \\
Teontepec & Puebla & 0.040 \\
Teteletitlán $(*)$ & Puebla & 0.044 \\
Texcala $(*)$ & Puebla & 0.228 \\
Zapotitlán $(*)$ & Puebla & 0.022 \\
Total & & 0.959 \\
\hline
\end{tabular}

$(*)$ Localidades visitadas en este estudio. $(\bullet)$ No se cuenta con información del área ocupada.

ejemplares existentes datan de 1958 a 1995 y proceden de 5 localidades del estado de Puebla (San Antonio Texcala, Nicolás Bravo, Tecamachalco, San Lucas Teteletitlán y Santiago Nopala).

De acuerdo con los cálculos de las distancias entre las 11 localidades, las poblaciones más cercanas son Texcala y Coapan separadas por $3.66 \mathrm{~km}$ (Cuadro 3). Tecamachalco resultó la más aislada con una distancia promedio de 52.60 km (Cuadro 3).

El área comprendida por el polígono que engloba las 11 poblaciones es aproximadamente de $2087 \mathrm{~km}^{2}$. Es poco probable que la información de las 8 localidades restantes incremente significativamente el área del polígono construido en este estudio. Más aún, el área real sumando las superficies ocupadas por las poblaciones es mucho menor. Para las 10 poblaciones estudiadas por Peters y Martorell (2000), el área estimada es tan sólo de $0.959 \mathrm{~km}^{2}$ (Cuadro 2). Debido a que estos 2 valores son inferiores al $5 \%$ de la superficie del territorio nacional (ca. 2 millones de $\mathrm{km}^{2}$ ), se asignó un valor de 4 (= muy restringida) en lo que se refiere a la amplitud de su distribución.

Criterio B), estado del hábitat respecto al desarrollo natural del taxón. El análisis de las características del ambiente físico de las 7 localidades indica que éstas se presentan en altitudes que van de 1778 a $2100 \mathrm{~m}$ snm, con pendientes de suaves a moderadas, suelos calizos de profundidad menor a $7.46 \mathrm{~cm}$ y alta pedregosidad superficial (Cuadro 4). Las laderas no presentaron una orientación en particular (Cuadro 4). Las poblaciones se encuentran dentro de matorrales xerófilos, pero la fisonomía de éstos cambia dependiendo de los vegetales dominantes (Cuadro 5). La cobertura vegetal de los sitios oscila de $20 \%$ al $73 \%$ 
Cuadro 3. Matriz de distancias entre poblaciones (km) de Mammillaria pectinifera

\begin{tabular}{cccccccccccc}
\hline Localidad & $A Z U$ & COA & $E R$ & FRO & NB & NOP & TEC & TEO & TET & TEX & ZAP \\
\hline AZU & ------- & 29.60 & 25.54 & 50.65 & 9.75 & 37.00 & 38.24 & 21.27 & 42.63 & 30.26 & 35.01 \\
COA & 29.60 & ------- & 5.97 & 30.45 & 29.22 & 26.54 & 59.36 & 14.32 & 22.60 & 3.66 & 6.97 \\
ER & 25.54 & 5.97 & ------ & 29.18 & 26.92 & 22.25 & 53.47 & 8.35 & 20.89 & 4.84 & 9.50 \\
FRO & 50.65 & 30.45 & 29.18 & ------ & 54.94 & 16.50 & 62.68 & 29.59 & 8.35 & 26.99 & 24.12 \\
NB & 9.75 & 29.22 & 26.92 & 54.94 & ------ & 43.16 & 47.54 & 25.60 & 46.64 & 31.00 & 35.69 \\
NOP & 37.00 & 26.54 & 22.25 & 16.50 & 43.16 & ------ & 46.45 & 18.12 & 11.92 & 23.02 & 23.22 \\
TEC & 38.24 & 59.36 & 53.47 & 62.68 & 47.54 & 46.45 & ------ & 45.25 & 57.98 & 57.83 & 57.22 \\
TEO & 21.27 & 14.32 & 8.35 & 29.59 & 25.60 & 18.12 & 45.25 & ------- & 21.43 & 12.58 & 16.44 \\
TET & 42.63 & 22.60 & 20.89 & 8.35 & 46.64 & 11.92 & 57.98 & 21.43 & ------ & 19.03 & 16.78 \\
TEX & 30.26 & 3.66 & 4.84 & 26.99 & 31.00 & 23.02 & 57.83 & 12.58 & 19.03 & ------ & 4.78 \\
ZAP & 35.01 & 6.97 & 9.5 & 24.12 & 35.69 & 23.22 & 57.22 & 16.44 & 16.78 & 4.78 & ------- \\
Media & 32.00 & 22.87 & 20.69 & 33.35 & 35.05 & 26.82 & 52.6 & 21.30 & 26.83 & 21.40 & 22.97 \\
EE & 3.64 & 5.24 & 4.66 & 5.48 & 4.21 & 3.66 & 2.47 & 3.31 & 5.19 & 5.25 & 5.09 \\
\hline
\end{tabular}

$A Z U=$ Azumbilla, $C O A=$ Coapan, $E R=$ El Riego, $F R O=$ Frontera, $N B=$ Nicolás Bravo, $N O P=$ Nopala, $T E C=$ Tecamachalco, $T E O=$ Teontepec, $T E T=$ Teteletitlán, $T E X=$ Texcala, $Z A P=$ Zapotitlán. $E E=$ error estándar

Cuadro 4. Características físicas de las 7 localidades de Mammillaria pectinifera visitadas en este estudio

\begin{tabular}{lcccccccc}
\hline Localidad & Estado & Altitud $(m$ snm) & Pendiente $\left(^{\circ}\right)$ & Orientación & \multicolumn{2}{c}{ Pedregosidad (\%) } & \multicolumn{2}{c}{ Profundidad del suelo (cm) } \\
& & & & & Media & EE & Media & EE \\
\hline Coapan & Puebla & 1778 & 5 & Este & 40 & 0.0 & 3.39 & 0.45 \\
El Riego & Puebla & 1905 & 10 & Noroeste & 68.33 & 1.67 & 6.78 & 1.15 \\
Frontera & Oaxaca & 1950 & 10 & Noroeste & 61.66 & 1.67 & 4.11 & 0.19 \\
Tecamachalco & Puebla & 2070 & 20 & Suroeste & 9 & 2.08 & 5.75 & 3.29 \\
Teteletitlán & Puebla & 2100 & 15 & Oeste & 76.66 & 1.67 & 7.46 & 0.82 \\
Texcala & Puebla & 1914 & 28 & Sureste & 73.33 & 1.67 & 2.66 & 0.84 \\
Zapotitlán & Puebla & 2130 & 12 & Oeste & 75 & 0.0 & 7.12 & 0.63 \\
\hline
\end{tabular}

$E E=$ error estándar

(Cuadro 5).

Estos resultados están apoyados en los de ZavalaHurtado y Valverde (2003). Mediante un análisis de los microambientes en la localidad de Texcala, los autores encontraron que $M$. pectinifera se ubica en comunidades caracterizadas por una baja diversidad vegetal, suelos alcalinos profundos con alta pedregosidad superficial y gran capacidad de retención de agua. En buena medida, la distribución restringida de $M$ pectinifera se relaciona con su alta especificidad de hábitat (Zavala-Hurtado y Valverde, 2003). Si bien no contamos con información sobre la disponibilidad de sitios adecuados para $M$. pectinifera, los registros sobre el número de poblaciones en la región sugieren que estos sitios son escasos. Por esta razón se asignó un valor de 2 (= intermedio o limitante) para este criterio.

Criterio C), vulnerabilidad biológica intrínseca del taxón. La densidad de $M$. pectinifera en las poblaciones estudiadas oscila entre 12.2 y $132.2 \mathrm{ind} / 100 \mathrm{~m}^{2}$ (Cuadro $6)$, con una media ( $\pm \mathrm{EE}$ ) para las 7 poblaciones de 56.7 $( \pm 17.5)$ ind./100 $\mathrm{m}^{2}$. El porcentaje de individuos con protección por vegetales o rocas varía de 4 a $41 \%$ y de 8 a $71 \%$, respectivamente, mientras que el correspondiente a los individuos sin protección fluctúa de 0 a $88 \%$ (Cuadro 6). En estudios previos no se encontró evidencia de que M. pectinifera establezca relaciones de nodricismo con 
Cuadro 5. Características de la comunidad vegetal en las 7 localidades de Mammillaria pectinifera evaluadas en este estudio

\begin{tabular}{|c|c|c|c|}
\hline Localidad & Cobertura vegetal (\%) & Especies dominantes & Familia \\
\hline Coapan & 35 & $\begin{array}{c}\text { Yucca periculosa } \mathrm{F} \text {. Baker } \\
\text { Hechtia podantha Mez. } \\
\text { Pedilantus cymbifera } \\
\text { Echinocactus platyacanthus Link et Otto } \\
\text { Agave peacockii Croucher } \\
\text { Acacia subangulata } \text { Rose } \\
\text { Agave marmorata } \text { Roezl } \\
\text { Ferocactus latispinus (Haw.) Britton et Rose } \\
\text { Bursera simaruba } \text { (L.) Sarg. } \\
\text { Coryphantha pallida } \text { Britton et Rose } \\
\text { Sanvitalia futicosa Hemsley } \\
\text { Especie no identificada } 1 \text { (Ocotillo) }\end{array}$ & $\begin{array}{l}\text { Agavaceae } \\
\text { Bromeliaceae } \\
\text { Euphobiaceae } \\
\text { Cactaceae } \\
\text { Agavaceae } \\
\text { Mimosaceae } \\
\text { Agavaceae } \\
\text { Cactaceae } \\
\text { Burseraceae } \\
\text { Cactaceae } \\
\text { Asteraceae } \\
\text {------ }\end{array}$ \\
\hline El Riego & 60 & $\begin{array}{c}\text { Acacia subangulata } \text { Rose } \\
\text { Calliandropsis nervosus (Britton et Rose) H. Hern et Guinet } \\
\text { Hechtia podantha Mez. } \\
\text { Lippia graveolens Kunth } \\
\text { Dasylirion acrotriche (Schiede) Zucc. } \\
\text { Echinocactus platyacanthus Link et Otto } \\
\text { Especie no identificada } 1 \text { (Ocotillo) }\end{array}$ & $\begin{array}{l}\text { Mimosaceae } \\
\text { Mimosaceae } \\
\text { Bromeliaceae } \\
\text { Verbenaceae } \\
\text { Nolinaceae } \\
\text { Cactaceae } \\
\text {------------ }\end{array}$ \\
\hline Frontera & 73 & $\begin{array}{c}\text { Agave kerchovei Lemm. } \\
\text { Calliandropsis nervosus (Britton et Rose) H. Hern et Guinet } \\
\text { Acacia subangulata } \text { Rose } \\
\text { Turnera difussa Willd ex Schult } \\
\text { Mimosa luisana } \text { Brandg. } \\
\text { Coryphantha pallida } \text { Britton et Rose } \\
\text { Especie no identificada } 2 \text { (compuesta 10) }\end{array}$ & $\begin{array}{l}\text { Agavaceae } \\
\text { Mimosaceae } \\
\text { Mimosaceae } \\
\text { Mimosaceae } \\
\text { Mimosaceae } \\
\text { Cactaceae } \\
\text { Asteraceae }\end{array}$ \\
\hline Tecamachalco & 20 & $\begin{array}{l}\text { Sanvitalia futicosa Hemsley } \\
\text { Sanvitalia procumbens Lam. } \\
\text { Opuntia pilifera Weber } \\
\text { Castela tortuosa Liebm. } \\
\text { Agave sp. } \\
\text { Ferocactus robustus (Pfeiffer) Britt. et Rose } \\
\text { Leucophyllum pringlei (Greenman) Standley } \\
\text { Especie no identificada 1 (Ocotillo) }\end{array}$ & $\begin{array}{l}\text { Asteraceae } \\
\text { Asteraceae } \\
\text { Cactaceae } \\
\text { Simaroubaceae } \\
\text { Agavaceae } \\
\text { Cactaceae } \\
\text { Scrophulariaceae } \\
\text {------- }\end{array}$ \\
\hline Teteletitlán & 30 & $\begin{array}{c}\text { Dasylirion acrotriche (Schiede) Zucc. } \\
\text { Agave kerchovei } \text { Lemm. } \\
\text { Mammillaria sphacelata Martius } \\
\text { Mimosa luisana } \text { Brandg. } \\
\text { Calliandropsis nervosus (Britton et Rose) H. Hern et Guinet }\end{array}$ & $\begin{array}{l}\text { Nolinaceae } \\
\text { Agavaceae } \\
\text { Cactaceae } \\
\text { Mimosaceae } \\
\text { Mimosaceae }\end{array}$ \\
\hline Texcala & 20 & $\begin{array}{c}\text { Cnidoscolous tehuacanensis Breckon } \\
\text { Gomphrena decumbens Jacq. } \\
\text { Calliandropsis nervosus (Britton et Rose) H. Hern et Guinet } \\
\text { Agave marmorata Roezl } \\
\text { Agave stricta Salm-Dyck } \\
\text { Agave potatorum Zucc. } \\
\text { Coryphantha pallida } \text { Britton et Rose } \\
\text { Mammillaria haageana } \text { Pfeiffer } \\
\text { Senna pringlei } \text { Rose }\end{array}$ & $\begin{array}{l}\text { Euphobiaceae } \\
\text { Amaranthaceae } \\
\text { Mimosaceae } \\
\text { Agavaceae } \\
\text { Agavaceae } \\
\text { Agavaceae } \\
\text { Cactaceae } \\
\text { Cactaceae } \\
\text { Caesalpiniaceae }\end{array}$ \\
\hline Zapotitlán & 60 & $\begin{array}{l}\text { Gymnosperma glutinosa } \text { Spreng } \\
\text { Sanvitalia fruticosa } \text { Hemsley } \\
\text { Dasylirion acrotriche (Schiede) Zucc. } \\
\text { Viguiera dentata (Cav.) Sprengel } \\
\text { Schaefferia stenophylla } \text { Standley } \\
\text { Acacia subangulata } \text { Rose }\end{array}$ & $\begin{array}{l}\text { Asteraceae } \\
\text { Asteraceae } \\
\text { Nolinaceae } \\
\text { Asteraceae } \\
\text { Celastraceae } \\
\text { Mimosaceae }\end{array}$ \\
\hline
\end{tabular}


Cuadro 6. Densidades poblacionales y ubicación de los individuos respecto a la protección o no de vegetales o rocas en las siete localidades de Mammillaria pectinifera. El valor entre paréntesis corresponde con el error estándar

\begin{tabular}{|c|c|c|c|c|}
\hline Localidad & $\begin{array}{l}\text { Densidad } \\
\left(\text { ind } / 100 m^{2}\right)\end{array}$ & $\begin{array}{c}\text { Individuos bajo } \\
\text { protección vegetal (\%) }\end{array}$ & $\begin{array}{c}\text { Individuos protegidos } \\
\text { por rocas }(\%)\end{array}$ & $\begin{array}{l}\text { Individuos sin } \\
\text { protección (\%) }\end{array}$ \\
\hline Coapan & $44.0(14.0)$ & $53.64(8.45)$ & $35.15(13.63)$ & $11.2(5.69)$ \\
\hline El Riego & $65.0(7.0)$ & $19.31(3.97)$ & $46.91(8.3)$ & $33.76(6.71)$ \\
\hline Frontera & $23.1(4.0)$ & $36.84(8.36)$ & $37.01(8.6)$ & $28.22(9.44)$ \\
\hline Tecamachalco & $12.2(6.0)$ & $40.47(30.39)$ & 0 & $59.52(30.3)$ \\
\hline Teteletitlán & $16.6(3.0)$ & 0 & $8.33(8.03)$ & $91.67(8.3)$ \\
\hline Texcala & $132.2(19.0)$ & $14.76(2.11)$ & $69.1(5.58)$ & $16.12(5.99)$ \\
\hline Zapotitlán & $103.8(43.0)$ & $2.97(1.81)$ & $10.74(6.33)$ & 86.28 (4.69) \\
\hline Media & $56.7(17.5)$ & $23.998(7.62)$ & $29.605(9.29)$ & $46.681(12.39)$ \\
\hline
\end{tabular}

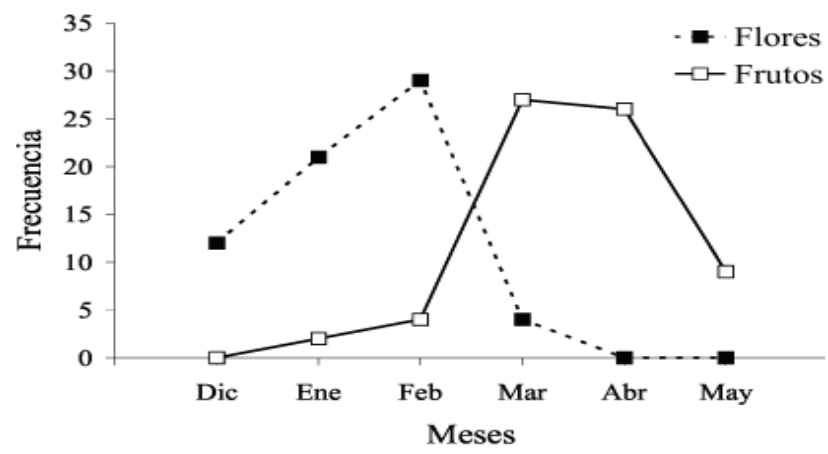

Figura 2. Patrones de floración y fructificación de Mammillaria pectinifera en la localidad de Texcala (Puebla) durante 1995 y 1996.

otras especies vegetales de la región (Rodríguez-Ortega y Ezcurra, 2000; Zavala-Hurtado y Valverde, 2002).

La floración y fructificación se concentra entre los meses de diciembre a mayo. La producción de flores inicia a finales del otoño (diciembre), continúa durante el invierno (enero-marzo), alcanzando un máximo en febrero (Fig. 2). Las flores de M. pectinifera permanecen alrededor de 2 días, la antesis comienza a las $0900 \mathrm{~h}$ termina alrededor de las $1300 \mathrm{~h}$, periodo durante el cual son visitadas por insectos. El número promedio de semillas por fruto estimado para las poblaciones de Texcala y Coapan fue de $11.80 \pm 1.29(n=27$ frutos $)$ y $16.07 \pm 1.23$ semillas $(n$ $=30$ frutos), respectivamente. Como en el caso de otras especies del género, los frutos maduran en el interior de los tallos, particularmente entre los tubérculos o mamilas (Bravo-Hollis y Sánchez-Mejorada, 1991). Este fenómeno conocido como serotinia (Lamont, 1991) implica que una fracción de las semillas permanece en la planta indefinidamente (Boke, 1960), lo cual podría representar una limitación en la dispersión en M. pectinifera (ZavalaHurtado y Valverde, 2003).
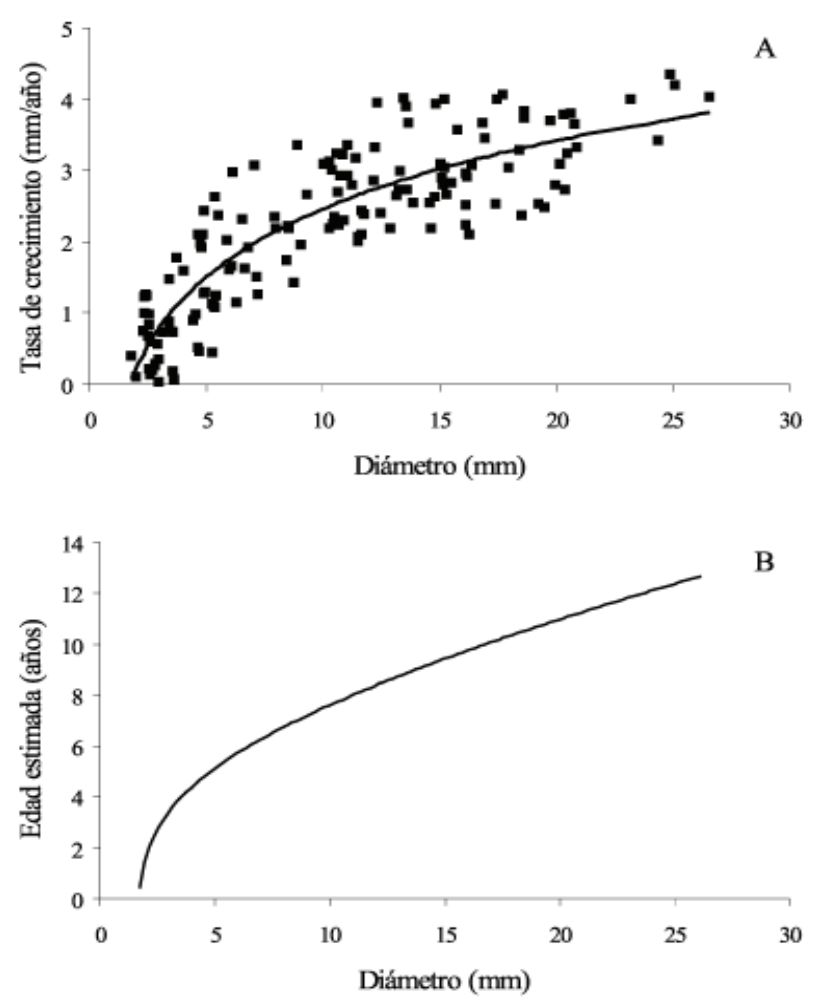

Figura 3. A, Relación entre el tamaño (diámetro) de los individuos registrados de Mammillaria pectinifera y la tasa de crecimiento observada (cuadrados) y estimada (línea continua) a partir del modelo de regresión $\left(r^{2}=0.761 ; p<0.0001\right)$; B, Relación entre el tamaño (diámetro) y la edad estimada a partir de la tasa de crecimiento estimada para individuos de M. pectinifera.

El crecimiento de los individuos de $M$. pectinifera es lento. El modelo obtenido $\left(r^{2}=0.761, p=0.0001\right.$, Fig. 3) revela que los individuos grandes presentan una tasa de crecimiento corporal mayor que los de menor talla. En la figura 3 se aprecia que la tasa de crecimiento tiende a 
estabilizarse alcanzando un valor asintótico de $4.0 \mathrm{~mm} /$ año. De acuerdo con esta información, las plántulas (0.01$2.5 \mathrm{~mm}$ ) tendrían de 0 a 3 años de edad y los juveniles (> 2.5-12 mm) de 3 a 8.45 años de edad. Los adultos pequeños (> 12-20 mm) podrían tener una edad de 8.43 a 11 años mientras los adultos mayores a $20 \mathrm{~mm}$ tendrían una edad superior a los 11 años. Estos resultados son apoyados por análisis del crecimiento temprano de $M$. pectinifera bajo condiciones controladas de humedad y radiación lumínica registrados por Martínez-Berdeja (2007). En dicho estudio, la estimación de la tasa relativa de crecimiento promediada entre tratamientos fue de $0.0106 \mathrm{~g} / \mathrm{g} / \mathrm{día}$. Esta tasa de crecimiento es, incluso, ligeramente más baja que las registradas para otras cactáceas (Martínez-Berdeja, 2007).

Según este modelo, la madurez reproductiva en los individuos de $M$. pectinifera se presenta cuando las plantas han alcanzado una talla mayor a $12 \mathrm{~mm}$ o una edad superior a los 8 años. Lo anterior contrasta con los registros de plantas propagadas bajo condiciones de invernadero, en donde la madurez reproductiva se alcanza 2 años después de la germinación (Anderson et al., 1994) y da una idea de los desafíos que afrontan los individuos de M. pectinifera en su ambiente natural (Valverde y ZavalaHurtado, 2006).

En la única población para la que se han registrado estudios de su dinámica, Valverde y Zavala-Hurtado (2006) estimaron una tasa de crecimiento poblacional menor a 1 (i.e., $\lambda=0.743$ ) con los datos de un año de observación. Cabe destacar que esta es la población de $M$. pectinifera con la mayor densidad. En el mismo estudio, tanto los análisis de elasticidad como las simulaciones realizadas modificando las probabilidades de transiciones, indican que la permanencia, principalmente de los juveniles y adultos, constituyen los procesos demográficos con mayor repercusión sobre los valores de $\lambda$.

Debido a la baja densidad, limitada dispersión (i.e., retención de frutos), lento crecimiento, edad a la primera reproducción (i.e., superior a los 8 años en condiciones naturales) y características demográficas (i.e., tasa de crecimiento poblacional menor a la unidad), se le asignó un valor de vulnerabilidad de 3 (= vulnerabilidad alta).

Criterio D) Impacto de la actividad humana sobre el taxón. De acuerdo con el índice de disturbio reescalado (ID $)$, los sitios menos perturbados, Zapotitlán y Pedernal, resultaron ser las localidades de más difícil acceso (Cuadro 7). Por el contrario, Tecamachalco y Coapan con los ID más altos, son las localidades que presentan el mayor grado de deterioro (Cuadro 7). La población de $M$. pectinifera del sitio Tecamachalco se ubica en una zona urbana, mientras que Coapan se encuentra cercana a un confinamiento de desechos sólidos.

El modelo que integra las variables consideradas para generar el índice de disturbio reescalado $\left(\mathrm{ID}_{r}\right)$ es:

$$
\begin{aligned}
& \mathrm{ID}_{r}=[0.568(\mathrm{CABR})-0.006(\mathrm{GANA})+0.587(\mathrm{RAMO}) \\
& +0.572(\mathrm{CAGA})+0.069(\mathrm{COMP})]+[-0.452(\mathrm{MACH}) \\
& +0.0(\mathrm{INCE})+0.471(\mathrm{CCHU})+0.442(\mathrm{POBL})+ \\
& 0.330(\mathrm{ADYA})+0.519(\mathrm{USOS})]+[-0.707(\mathrm{EROS})+ \\
& 0.707(\mathrm{ISLA})+0.0(\mathrm{STOM})]
\end{aligned}
$$

En el modelo se indican, entre corchetes, los tres agentes de disturbio (ganadería, actividades humanas y deterioro del hábitat, en ese orden) y entre paréntesis a los 14 indicadores (en el mismo orden del Cuadro 1). En el caso de la ganadería, la contribución principal al $\mathrm{ID}_{\mathrm{r}}$ está dada por variables relacionadas con el pastoreo extensivo de chivos. Respecto a las actividades humanas, destaca la contribución del cambio en el uso del suelo (USOS) y la ausencia de evidencia de incendios (INCE). Finalmente, en cuanto al deterioro del hábitat, sólo las variables EROS e ISLA con coeficientes relativamente altos, tuvieron una contribución importante al $\mathrm{ID}_{\mathrm{r}}$.

El patrón de densidad de $M$. pectinifera observado y el ajustado a una distribución de Poisson mediante una regresión logarítmica $(p<0.00001)$ muestra que la máxima

Cuadro 7. Sumatoria de los puntajes del ACP para cada uno de los 3 agentes de disturbio considerados (ganadería, actividades humanas y deterioro del hábitat), índice de disturbio (ID, sumatoria de los puntajes de los 3 agentes de disturbio) e índice de disturbio reescalado $\left(\mathrm{ID}_{\mathrm{r}}\right)$ para las 7 poblaciones de Mammillaria pectinifera estudiadas. Las poblaciones se enlistan en orden ascendente respecto al ID $_{\mathrm{r}}$

\begin{tabular}{lccccc}
\hline \multicolumn{1}{c}{ Localidad } & Ganadería & Actividades humanas & Deterioro del hábitat & $I D$ & $I D_{r}$ \\
\hline Zapotitlán & -2.394 & -2.010 & -2.073 & -6.478 & 0.000 \\
Frontera & -1.133 & -3.628 & -1.686 & -6.448 & 0.394 \\
El Riego & -0.891 & -1.902 & -2.073 & -4.866 & 21.214 \\
Teteletitlán & -0.369 & -1.025 & -2.073 & -3.466 & 39.637 \\
Texcala & 1.397 & -1.837 & -2.073 & -2.513 & 52.186 \\
Coapan & -0.050 & 0.633 & -0.138 & 0.445 & 91.114 \\
Tecamachalco & 2.696 & -0.470 & -1.106 & 1.120 & 100.000 \\
\hline
\end{tabular}


abundancia se presenta en condiciones con valores de disturbio intermedios (entre 45 y 55, Fig. 4). La densidad más alta $\left(132.22\right.$ individuos $\left./ 100 \mathrm{~m}^{2}\right)$ se encontró en Texcala con un índice de disturbio intermedio (52.186), seguida por la población de Zapotitlán (103.89 inds./100 m²) la cual presentó el índice de disturbio más bajo (0.00). Por otra parte, la población de Tecamachalco, con la densidad más baja (12.22 inds./100 $\mathrm{m}^{2}$ ), presentó el más alto índice de disturbio (100.00), mientras que la población de Frontera con una densidad de 23.11 inds. $/ 100 \mathrm{~m}^{2}$, mostró el segundo índice de disturbio más bajo (0.394).

Pese a que en éstas como en la mayoría de las localidades de $M$. pectinifera se presentan el impacto del pastoreo extensivo de chivos, el cambio en el uso del suelo y el deterioro del hábitat, las poblaciones con mayor densidad se encuentran en ambientes con índices de disturbio moderados (Fig. 4). Estos resultados son consistentes con los registrados por Martorell y Peters (2005), por lo que se le asignó un valor de impacto de 3 (= impacto medio) en este criterio.

\section{Discusión}

Recientemente, los esfuerzos de conservación se han orientado a salvaguardar ecosistemas, comunidades, biotas o paisajes (Franklin, 1993; Delcourt y Delcourt, 1998; Cameron, 2002). El razonamiento detrás de este esfuerzo es la importancia de conservar tales unidades para preservar procesos ecológicos, hábitats, interacciones bióticas o hotspots (Franklin, 1993; Mills et al, 1993; Orians, 1993; Reid, 1998; Thompson, 1998). Sin embargo, los enfoques sobre especies o poblaciones también son relevantes para establecer prioridades de conservación, particularmente de

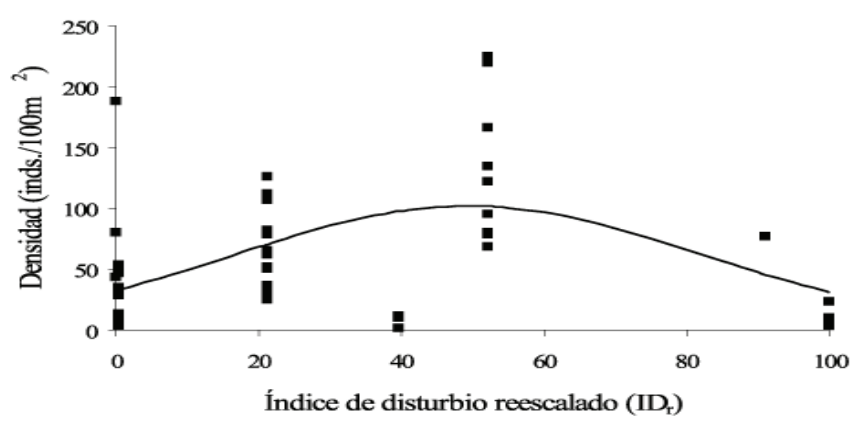

Figura 4. Densidad de Mammillaria pectinifera como respuesta al índice de disturbio reescalado (ID) estimado. La línea continua indica el ajuste a un modelo lineal generalizado con distribución de Poisson y función de enlace logarítmica $(p<0.00001)$ y los cuadrados indican los valores observados en las 7 poblaciones estudiadas. especies vulnerables, raras o amenazadas (e.g., Schemske et al., 1994; Wiser et al., 1998; Sutherland, 2000; Hedrick, 2001; van Tienderen et al., 2002), y el MER representa una metodología puntual para identificar especies silvestres vulnerables en México. Este protocolo evalúa el riesgo de extinción documentando y sistematizando los factores que afectan a cualquier especie silvestre en el territorio nacional (Tambutti et al., 2001). El MER no privilegia un criterio o enfoque en particular, sino que se basa en la evaluación cualitativa de cuatro criterios para lograr la mayor objetividad posible en la toma de decisiones. Partiendo de que el MER no asigna valores éticos o de conservación a ninguna especie (Tambutti et al., 2001), la evaluación del riesgo de extinción podrá ser realizada en cada especie silvestre presente en México.

De acuerdo con la evaluación de los 4 criterios independientes establecidos en el MER, la sumatoria obtenida es de 12 puntos $(=4+2+3+3)$, por lo que este trabajo propone que $M$. pectinifera debe cambiar de categoría en la Norma Oficial Mexicana NOM-059ECOL-2001, de especie amenazada (A) a especie en peligro de extinción (P) (de 12 a 14 puntos, SEMARNAT, 2002). No obstante que $M$. pectinifera permanece en la lista del Apéndice I de la CITES (CITES, 2007), ya no es considerada como especie amenazada en el listado de la Unión Internacional para la Conservación de la Naturaleza (UICN) (Guzmán et al., 2003; IUCN, 2007). La exclusión obedece a que $M$. pectinifera no ha sido revaluada con los criterios vigentes de la Lista Roja (versión 3.1) adoptados desde 2001 (Héctor M. Hernández, comunicación personal). Si bien no existe equivalencia exacta entre las categorías de riesgo del MER y la UICN (Tambutti et al., 2001), la categoría de especie en peligro de extinción de la Norma Oficial Mexicana coincide parcialmente con las categorías en peligro crítico (CR) y en peligro (EN) de la clasificación de la UICN (SEMARNAT, 2002; Arias et al., 2005). Los resultados de la evaluación realizada en este estudio sugieren que $M$. pectinifera debe ser considerada nuevamente en la Lista Roja de la UICN con la categoría de especie en peligro crítico (CR), categoría también equivalente al Apéndice I de la CITES (Arias et al., 2005).

El MER incorpora información de distintas fuentes y su confiabilidad dependerá, en mucho, de la calidad de los datos obtenidos. En el presente estudio, las decisiones sobre la calificación asignada a cada uno de los criterios del MER aplicados a $M$. pectinifera se documentaron y argumentaron con base en la mejor información disponible. Es indudable que los valores asignados a uno o más de los criterios del MER son susceptibles de modificación a la luz de los resultados que aporten futuras investigaciones; no obstante, esto no implicaría necesariamente un cambio substancial en el estatus de conservación de $M$. pectinifera. Así, por ejemplo, la incorporación de nuevas localidades de $M$. pectinifera difícilmente modificaría el valor del 
Criterio A referente a la amplitud del área de distribución. De hecho, la estimación más optimista, es decir 2087 km², representa tan solo el $0.10 \%$ del territorio nacional.

Los estudios sobre la biología y ecología de $M$. pectinifera son escasos. La asignación de un valor intermedio para el Criterio B resultó de reconocer que, si bien, la condición del hábitat donde se establece $M$. pectinifera no es hostil, la frecuencia de éstos dentro del rango de distribución de la especie parece ser baja (ZavalaHurtado y Valverde, 2003). Aunque los datos con los que se cuenta sugieren que los requerimientos particulares de M. pectinifera la limitan a ciertos parches adecuados del hábitat, otros factores inherentes a su biología podrían estar relacionados con su vulnerabilidad (Zavala-Hurtado y Valverde, 2003). Al igual que la gran mayoría de las cactáceas, $M$. pectinifera presenta atributos tales como limitada capacidad de dispersión, bajo reclutamiento, tasa de crecimiento poblacional negativa y lento crecimiento de los individuos, entre otros. Mammillaria pectinifera es una especie con alta vulnerabilidad biológica y la asignación de la máxima calificación en el Criterio $\mathrm{C}$ refleja esta situación.

Tal vez para el Criterio D, que se refiere al impacto de las actividades humanas, es para el que la SEMARNAT (2002) aporta la menor cantidad de elementos para su evaluación. Por esta razón, se optó por aplicar el método de Martorell y Peters (2005) que por medio del índice ID permite medir el grado de disturbio e identificar cuál de los agentes de perturbación tiene el mayor impacto (i.e., ganadería, actividades humanas y deterioro del hábitat). Por ejemplo, las poblaciones con los valores más altos de $\mathrm{ID}_{\mathrm{r}}$ (i.e., Tecamachalco y Coapan) son aquellas que enfrentan el mayor grado de disturbio y éstos presentan valores altos en uno o varios de los indicadores de perturbación. Las localidades mencionadas presentaron el primero o segundo más alto puntaje para al menos uno de los 3 indicadores (véase Cuadro 7). Ambas poblaciones padecen fuertes presiones como consecuencia de la urbanización y del cambio en el uso del suelo. Sin embargo, el análisis del efecto de la intensidad del disturbio ( ID $_{\mathrm{r}}$ ) sobre la densidad mostró que la máxima densidad se presenta en sitios con valores de disturbio intermedios, lo que revela que $M$. pectinifera es tolerante a ciertos niveles de disturbio y sugiere un cierto grado de ruderalidad (Peters y Martorell, 2000; Martorell y Peters, 2005). Así, la calificación de impacto medio en este criterio del MER refleja el hecho de que las máximas densidades de $M$. pectinifera se presentaron bajo condiciones de disturbio moderado.

No obstante las virtudes del MER, el ejercicio de evaluación no está exento de problemas de interpretación, subjetividad o carencia de independencia entre los criterios (Tambutti et al., 2001; Olson et al., 2005). Incluso la falta de información puede vulnerar la aplicación de los criterios del MER a muchas especies que han recibido poca atención. Olson y colaboradores (2005) han aportado sugerencias para mejorar la objetividad y validez del MER. Del presente estudio surge la propuesta de incorporar la metodología de Martorell y Peters (2005) en la valoración del Criterio D del MER. Aunque un factor a considerar en este criterio es el tráfico del taxón (SEMARNAT, 2002), que no está incluido en el índice de disturbio por la dificultad que representa su comprobación en el campo (Peters y Martorell, 2000). Sin embargo, esta propuesta metodológica permitirá evaluar adecuadamente el grado, naturaleza y efectos del disturbio sobre las poblaciones de plantas mexicanas en riesgo.

La evaluación del MER presentada en este estudio puede utilizarse en la generación de propuestas para la protección y conservación de $M$. pectinifera. Por ejemplo, a partir del reconocimiento de que $M$. pectinifera es una especie en peligro de extinción, es necesario establecer sistemas de monitoreo de poblaciones selectas de esta especie (Gaines et al., 1999). Esta selección de poblaciones se realizaría con base en el análisis de la estructura genética de cada población con el fin de monitorear y proteger aquellas donde se presente la mayor variabilidad genética o estructuras genéticas idiosincrásicas. Así también, el MER puede utilizarse para plantear hipótesis a probar en estudios posteriores. En este sentido, futuros estudios sobre aspectos básicos de la ecología y genética de $M$. pectinifera permitirán identificar aquellos factores que ponen en riesgo la permanencia de sus poblaciones.

\section{Agradecimientos}

Este estudio fue posible gracias al respaldo de la Universidad Autónoma Metropolitana-Iztapalapa y al financiamiento del proyecto FOSEMARNAT-2004-0126. Agradecemos el apoyo brindado por el Personal de la Reserva de la Biosfera Tehuacán-Cuicatlán así como el que recibimos de Pedro G. Miranda Pacheco para la realización de diversas fases de este proyecto.

\section{Literatura citada}

Anderson, E. F., S. Arias y N. P. Taylor. 1994. Threatened cacti of Mexico. Royal Botanic Gardens, Kew, London. 136 p.

Arias S., S. Gama y U. Guzmán. 1997. Las cactáceas del valle de Tehuacán-Cuicatlán, Flora del Valle de Tehuacán-Cuicatlán. Fascículo 14. Instituto de Biología, UNAM, México, D. F. $146 \mathrm{p}$.

Arias, S., U. Guzmán, M. C. Mandujano, M. Soto Galván, y J. Golubov. 2005. Las especies mexicanas de cactáceas en 
riesgo de extinción. I. Una comparación entre los listados NOM-059-ECOL-2001 (México), La Lista Roja (UICN) y CITES. Cactáceas y Suculentas Mexicanas 50:100-125.

Boke, N. H., 1960. Anatomy and development in Solisia. American Journal of Botany 47:59-65.

Bravo-Hollis, H. 1978. Las cactáceas de México, vol. I. Universidad Nacional Autónoma de México, México, D. F. $743 \mathrm{p}$.

Bravo-Hollis, H. y H. Sánchez-Mejorada. 1991. Las cactáceas de México, vol. II. Instituto de Biología, UNAM, México, D. F. 404 p.

Cameron, T. 2002. 2002: the year of the 'diversity-ecosystem function' debate. Trends in Ecology and Evolution 17:495496.

CITES. 2007. Appendix I. http://www.cites.org/eng/app/ appendices.shtlm Consultada: noviembre 2007.

Crawley, M. J. 1996. GLIM for ecologists.Blackwell Science. Oxford. 377 p.

Dávila, A. P., R. M. Lemos, A. Ramírez, A. Salinas y P. Tenorio. 1995. Análisis de la flora del valle de Tehuacán-Cuicatlán: endemismos y diversidad. In Conservación de plantas en peligro de extinción: diferentes enfoques, E. Linares, P. Dávila, F. Chiang, R. Bye y T. Elías (eds.). Instituto de Biología, UNAM, México, D. F. p. 133-41.

Delcourt, P. A y H. R. Delcourt. 1998. Paleoecological insights on conservation of biodiversity: a focus on species, ecosystems, and landscapes. Ecological Applications 8:921-934.

Franklin, J. F. 1993. Preserving biodiversity: species, ecosystems, or landscapes. Ecological Applications 3:202-205.

Gaines, W.L., R.J. Harrod y J.F. Lehmkuhl. 1999. Monitoring biodiversity: quantification and interpretation. General Technical Report PNW-GTR-443. USDA Forest Service, Portland, Oregon.

García, E. 1973. Modificaciones al sistema de clasificación climática de Köeppen. Instituto de Geografía, UNAM, México, D. F. 246 p.

Glass, C. 1998. Guía para la identificación de Cactáceas amenazadas de México. Vol. 1. Comisión Nacional para el Estudio de la Biodiversidad/Cante, México, D. F. 210 p.

Godínez-Álvarez, H., A. Valiente-Banuet y L. Valiente-Banuet. 1999. Biotic interactions and the population dynamics of the long-lived columnar cactus Neobuxbaumia tetetzo in the Tehuacán Valley, Mexico. Canadian Journal of Botany $77: 1-6$.

Guzmán, U., Arias, S., Dávila, P. 2003. Catálogo de cactáceas mexicanas. Universidad Nacional Autónoma de México/ Comisión Nacional para el Conocimiento y Uso de la Biodiversidad, México, D. F. 315 p.

Hedrick, P. W. 2001. Conservation genetics: where are we now? Trend in Ecology and Evolution 16:629-636.

Hernández, H. M. y H. Godínez. 1994. Contribución al conocimiento de las cactáceas mexicanas amenazadas. Acta Botanica Mexicana 26:33-52.

Hunt, D. 1992. CITES Cactaceae checklist. Royal Botanical Gardens, Kew. 190 p.

IUCN. 2007. IUCN Red List of threatened species. http://www. iucnredlist.org Consultada: noviembre 2007.

Kovach, W.L. 2004. MVSP-A multivariate statistical package for
Windows, ver. 3.12g. Kovach Computer Services, Pentraeth, Wales, $133 \mathrm{p}$.

Lamont, B. B. 1991. Canopy seed storage and release- what's in a name?. Oikos 60:266-268.

Martínez-Berdeja, A. 2007. Análisis de crecimiento temprano de tres cactáceas amenazadas (Mammillaria pectinifera, Obregonia denegrii y Coryphantha werdermannii) bajo condiciones controladas de humedad y radiación lumínica. Tesis, Facultad de Ciencias, UNAM, México, D. F. 90 p.

Martorell, C. y E. Peters. 2005. The measurement of chronic disturbance and its effects on the threatened cactus Mammillaria pectinifera. Biological Conservation 124:199207.

Meyrán, G. J. 1973. Guía botánica de cactáceas y otras suculentas del valle de Tehuacán. Sociedad Mexicana de Cactología A.C., México, D. F. 50 p.

Mills, L.S., M. E. Soulé y D. F. Doak. 1993. The keystonespecies concept in ecology and conservation. Biosience 43:219-224.

Olson, M. E., J. A. Lomelí y N. I. Cacho. 2005. Extinction threat in the Pedilanthus clade (Euphorbia, Euphorbiaceae), with special reference to the recently rediscovered E. conzattii ( $P$. pulchellus). American Journal of Botany 92:634-641.

Orians, G. 1993. Endangered at what level?. Ecological Applications 3:206-208.

Peters, E. y C. Martorell. 2000. Conocimiento y conservación de las mamilarias endémicas del Valle de Tehuacán-Cuicatlán. Reporte final del proyecto R166-CONABIO. México, D.F. $38 \mathrm{p}$.

Reid, W. V. 1998. Biodiversity hotspots. Trends in Ecology and Evolution 13:275-280.

Rodríguez-Ortega, C.E. y E. Ezcurra. 2000. Distribución espacial en el hábitat de Mammillaria pectinifera y M. carnea en el valle de Zapotitlán Salinas, Puebla, México. Cactáceas y Suculentas Mexicanas 45:4-14.

Rzedowski, J. 1988. Vegetación de México. Limusa, México, D.F. 432 p.

Schemske, D. W., B. C. Husband, M. H. Ruckelshaus, C. Goodwillie, I. M. Parker y J. G. Bishop. 1994. Evaluating approaches to the conservation of rare and endangered plants. Ecology 75:584-606.

SEMARNAT. 2002. (Secretaria de Medio Ambiente y Recursos Naturales). NOM-059-ECOL-2001. Protección ambientalEspecies nativas de México de flora y fauna silvestresCategorías de riesgo y especificaciones para su inclusión, exclusión o cambio-Lista de especies en riesgo. Diario Oficial de la Federación, segunda sección, 6 de marzo de 2002, 1-81.

Sutherland, W. J. 2000. The conservation handbook: research, management and policy. Blackwell Science, Oxford. 278 p.

Tambutti, M., A. Aldama, O. Sánchez, R. Medellín y J. Soberón. 2001. La determinación del riesgo de extinción de especies silvestres en México. Gaceta Ecológica 61:11-21.

Thompson, J. N. 1998. Evolutionary ecology and the conservation of biodiversity. Trends in Ecology and Evolution 11:300303.

Valverde, P. L. y J. A. Zavala-Hurtado. 2006. Assessing the ecological status of Mammillaria pectinifera Weber 
(Cactaceae), a rare and threatened species endemic of the Tehuacán-Cuicatlán Region in Central Mexico. Journal of Arid Environments 64:193-208.

van Tienderen, P. H., A. A. de Haan, C. G. van der Linden y B. Vosean. 2002. Biodiversity assessment using markers for ecologically important traits. Trends in Ecology and Evolution 17:577-582.

Vite, F., J. A. Zavala-Hurtado, M. A. Armella y M. D García-Suárez. 1992. Regionalización y caracterización macroclimática del matorral xerófilo. Superficies de respuesta a variables climáticas de once géneros de plantas característicos de este tipo de vegetación. Carta escala 1:8'000,000. Atlas Nacional de México. Instituto de Geografía, UNAP. S. White. 1998. Prediction of rare-plant ocurrence: a southern Appalachian example. Ecological Applications 8:909-920.

Zavala-Hurtado, J. A. 1997. Estatus ecológico de Mammillaria pectinifera Weber y Pachycereus fulviceps en el valle de Zapotitlán, Puebla. Reporte final del proyecto G022CONABIO. México, D. F. 94 p.

Zavala-Hurtado, J. A. y G. Hernández-Cárdenas. 1999. Estudio de caracterización y diagnóstico del área propuesta como Reserva de la Biosfera Tehuacán-Cuicatlán. Instituto Nacional de Ecología, SEMARNAP/Universidad Autónoma Metropolitana-Iztapalapa, México, D. F. 132 p.

Zavala-Hurtado, J. A. y P. L. Valverde, P. L. 2002. Restricción del hábitat en Mammillaria pectinifera Weber (Cactaceae), una especie amenazada, endémica del valle de Tehuacán, en el trópico mexicano. In Memorias III Simposio Internacional sobre la Flora Silvestre en Zonas Áridas, D. Vásquez del Castillo, M. Ortega Nieblas y R. A. Castillo Gámez (eds.). Universidad de Sonora, Hermosillo, Sonora, México. p. 231-241.

Zavala-Hurtado, J. A. y P. L. Valverde. 2003. Habitat restriction in Mammillaria pectinifera, a threatened endemic Mexican cactus. Journal of Vegetation Science 14:891-898. 